\title{
Evaluation of Semantic Interference Detection in Parallel Changes: an Exploratory Experiment
}

\author{
Danhua Shao, Sarfraz Khurshid and Dewayne E Perry \\ Electrical and Computer Engineering, The University of Texas at Austin \\ \{dshao, khurshid, perry\}@ece.utexas.edu
}

\begin{abstract}
Parallel developments are becoming increasingly prevalent in the building and evolution of large-scale software systems. Our previous studies of a large industrial project showed that there was a linear correlation between the degree of parallelism and the likelihood of defects in the changes. To further study the relationship between parallel changes and faults, we have designed and implemented an algorithm to detect "direct" semantic interference between parallel changes. To evaluate the analyzer's effectiveness in fault prediction, we designed an experiment in the context of an industrial project. We first mine the change and version management repositories to find sample versions sets of different degrees of parallelism. We investigate the interference between the versions with our analyzer. We then mine the change and version repositories to find out what faults were discovered subsequent to the analyzed interfering versions. We use the match rate between semantic interference and faults to evaluate the effectiveness of the analyzer in predicting faults. Our contributions in this evaluative empirical study are twofold. First, we evaluate the semantic interference analyzer and show that it is effective in predicting faults (based on "direct" semantic interference detection) in changes made within a short time period. Second, the design of our experiment is itself a significant contribution and exemplifies how to mine software repositories rather than use artificial cases for rigorous experimental evaluations.
\end{abstract}

\section{Introduction}

Parallel development has become a common phenomenon in the development of large-scale software systems. Multiple developers work on the same module or program at the same time. The need for parallel development has come about for a variety of reasons:

- the size of the software systems,
- time to market also brings pressure to develop new features or new products in a very short time,

- code ownership management is too expensive,

- the increase of globalization, and

- the geographical distribution of developers.

While parallel development increases productivity, it also causes problems. When developers work in parallel, it is likely that their changes may unintentionally interfere with each other.

In our earlier work [12] [13], we delineated the phenomena of, and the problems related to, parallel changes. In a subsystem of Lucent Technologies' 5ESS ${ }^{\text {TM }}$ Telephone Switching System, high degrees of parallelism happened at multiple levels. To disclose the relationship between parallel changes and faults, we studied prima facie conflicts at the textual level, checking the overlap between the lines changed by different developers. We found two important results: 1) $3 \%$ of the changes made within 24 hours by different developers physically overlapped each others' changes; and 2) there was a linear correlation between the degree of parallelism and the likelihood of a defect in the changes.

Our initial investigations focused on conflicts at the explicit syntactic level. We believe that there are more conflicts at the semantic level. To explore this hypothesis, we designed a semantic interference detection algorithm [21] [22], based on data dependency analysis and program slicing.

To investigate our hypothesis as well as the effectiveness and efficiency of our algorithm, we built SCA (Semantic Conflict Analyzer) and designed a rigorous empirical study to evaluate it in the same industrial context as our previous empirical studies.

In Section 2, we give an overview of the semantic interference detection algorithm. The context for this study is discussed in Section 3. Section 4 presents the experimental design and its results. We discuss validity issues in Section 5, and compare our work to related 
research in Section 6. Finally, we summarize our study and propose future work in Section 7.

\section{Overview of Semantic Interference}

Our semantic interference detection algorithm [21][22] combines data dependency analysis and program slicing. The data dependency analysis discloses the semantic structure of the program while the program slicing identifies semantic structures impacted by changes. By comparing the overlap of the impacted parts of the two versions, we can learn if they are in conflict.

\subsection{Semantic Analysis of Change Impact}

Semantic analysis of change impact is the basis for the semantic interference detection algorithm. Semantic program analysis discloses internal dependencies within programs. As a lightweight static analyzer, SCA only focuses on the local (i.e., intraprocedural) data flow dependencies related to variable def-use pairs. Figure 1 illustrates the semantic analysis of change impact.

First, SCA analyzes the semantic dependencies in the two versions. We use a triple (var: def, use) to represent a dependency, where var is the variable on which the dependence is built, def is the line that defines variable var, and the use line uses the variable defined at def line. The dependences in version $\mathrm{v} 1$ are $\{(\mathrm{a}: 1,3),(\mathrm{b}: 2,4),(\mathrm{i}: 3,5),(\mathrm{j}: 4,5)\}$.

With the variable use-def dependency analysis on the two versions, SCA calculates the change impact by forward slicing from the changed statements. In this example, the change from v1 to v2 modified Line 1 from " $\mathrm{a}=0$ " to " $\mathrm{a}=1$ ". According to the variable defuse chains, $\{(\mathrm{a}: 1,3)$, (i: 3, 5) $\}$, Line 3 and 5 are impacted. The impact of this change $\mathrm{v} 1 \rightarrow \mathrm{v} 2$ is $\{3,5\}$.

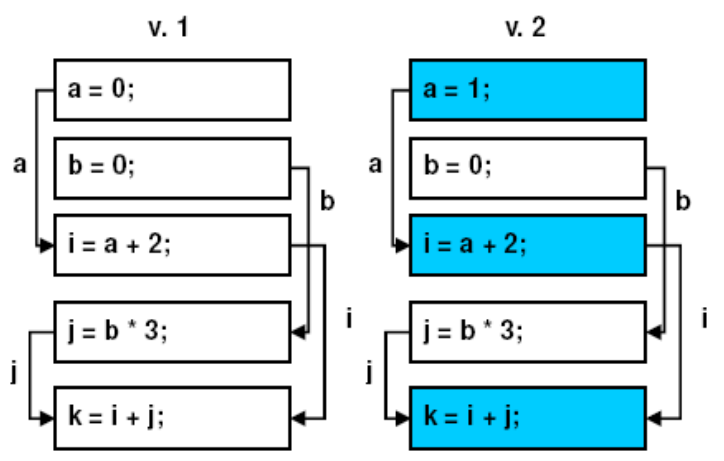

Figure 1 Semantic analysis on change: $v 1 \rightarrow \mathrm{v} 2$.

\subsection{Semantic Interference Detection}

In general, given two changes to be checked, we calculate the impact of each change according to the variable def-use dependencies. After mapping the impacted fragments of the two changes onto the final version, we can determine their interference by checking their overlaps. The detailed explanation is in [21] and [22].

Figure 2 illustrates the semantic interference detection algorithm. Suppose there are two adjacent changes: $\mathrm{v} 1 \rightarrow \mathrm{v} 2$ and $\mathrm{v} 2 \rightarrow \mathrm{v} 3$.

1) For each version, calculate data dependence graph and identify variable def-use pair. The results are: for $\mathrm{v} 1$, the dependency is $\{(\mathrm{a}: 1,3)$, $(\mathrm{b}$ : 2, 4), (i: 3, 5), (j: 4, 5) \}; version v2 is $\{(\mathrm{a}: 1,3)$, (b: 2, 4), (i: 3, 5), $(\mathrm{j}: 4,5)\}$, and version v3 is $\{(\mathrm{a}:$ 1, 3), (b: 2, 4), (i: 3, 5), (j: 4, 5)\};

2) For each change, identify the changed lines. In change $\mathrm{v} 1 \rightarrow \mathrm{v} 2$, Line 1 was changed and in change v2 $\rightarrow \mathrm{v} 3$, Line 2 was changed,

3) Calculate the semantic impact of the two changes by forward slicing from the changed lines. So, Impact $(\mathrm{v} 1 \rightarrow \mathrm{v} 2)=\{3,5\}$ and Impact $(\mathrm{v} 2 \rightarrow \mathrm{v} 3)=$ $\{4,5\}$;

4) Compare impacted lines of the two changes. Line 5 , where change $(\mathrm{v} 1 \rightarrow \mathrm{v} 2)$ and $(\mathrm{v} 2 \rightarrow \mathrm{v} 3)$ overlap with each other, is their semantic interferences.

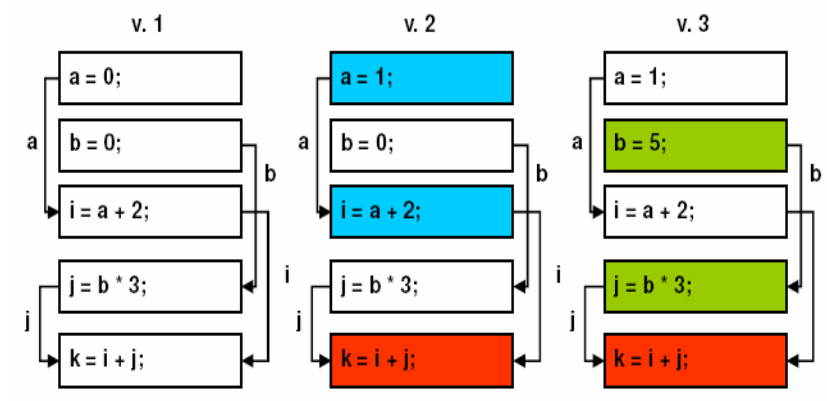

Figure 2 Detect semantic interference between changes: $\mathrm{v} 1 \rightarrow \mathrm{v} 2$ and $\mathrm{v} 2 \rightarrow \mathrm{v} 3$.

\section{Study Context}

In this study, the data repository from our previous study constitutes the base environment in which to evaluate our semantic interference detection algorithm.

\subsection{Change \& Version Mgmt Repositories}

This study is based on one of the 50 subsystems of $5 \mathrm{ESS}^{\mathrm{TM}}$, a successful industrial project with high degrees of parallel changes. 5ESS is a telephone switch project developed by Lucent Technologies [8] and has about 100,000,000 lines of $\mathrm{C}$ and $\mathrm{C}++$ code and 
another 100,000,000 lines in header files and makefiles. Its project structure (feature development) contributed to the high degree of parallel changes during the development process. In this subsystem of approximately 1.5 million lines of code, the number of developers reached 200 at its peak and dropped to a low of 50. Two products, one for US and one for international customers, were developed separately although some files are common for both of them.

The version and fault history data for our study comes from the change management system of $5 \mathrm{ESS}^{\mathrm{TM}}$. In Lucent Technologies, the evolution of $5 \mathrm{ESS}^{\mathrm{TM}}$ is managed by a two-layered system: a change management layer, ECMS [23], to initiate and track changes to the product, and a configuration management layer, SCCS [16], to manage the versions of files needed to construct the appropriate configurations of the product. In $5 \mathrm{ESS}^{\mathrm{TM}}$, the changes are recorded in a layered hierarchy: Feature, Initial Modification Request (IMR), Modification Request (MR) and delta. A feature is the fundamental unit of extension to the system, and each feature is composed of a set of IMRs that represent problems to be solved. All changes are handled by ECMS and are initiated using an IMR, which may have one or more MRs (each of which represents a solution, or part of a solution, to an IMR's problem), whether the change is for fixing a fault, perfecting or improving some aspect of the system, or adding new features to the system. Each functionally distinct set of changes to the code made by a developer is recorded as a MR by ECMS. For each $\mathrm{MR}$, there is a short abstract written by developers describe its purpose. We use the approach in [9] to classify MRs into according to their purposes: adaptive, perfective, and corrective. When a change is made to a file in the context of an MR, SCCS keeps track of the actual lines added, changed, or deleted. This set of changes is known as a delta. For each delta, ECMS records its date, the developer who made it, and the MR to which it belongs. So, from ECMS and SCCS, we can get both the actual changes on the source code and the purpose for the changes.

SCCS is a pessimistic version control system. At a given time only one developer can check out and modify a program. Changes representing different MRs are often interleaved with each other, providing a sequential set of changes but which represent logically parallel changes. We extend our definition of logically parallel changes further to include those changes made independently and committed by different developers within a short time interval.

\subsection{Parallel Changes in the Repository}

We chose this 5 ESS $^{\mathrm{TM}}$ subsystem to evaluate our SCA to provide continuity with our previous studies [12] [13], where we found the following:

- There are multiple levels of parallel development. Each day, there is ongoing work on multiple MRs by different developers solving different IMRs belonging to different features within different releases of two similar products aimed at distinct markets.

- The activities within each of these levels cut across common files. $12.5 \%$ of all deltas are made by different developers to the same files within a day of each other and some of these deltas interfere with each other.

- Over the interval of a particular release, the number of files changed by multiple MRs is $60 \%$ that are concurrent with respect to that release. These parallel MRs may result in interfering changes - though we would expect the degree of awareness of the implications of these changes to be higher than those made within one day of each other.

Furthermore, our previous study also found that there is a significant correlation between files with a high degree of parallel development and the number of faults. Using PCmax, the maximum number of parallel MRs per file in a day, as the measure of the degree of parallel changes, our analysis showed that high degrees of parallel changes tend to have more faults. The analysis of variance strongly indicates that, even accounting for the faults correlated with lifetime, size and numbers of deltas, parallel changes were a significant factor $(\mathrm{p}<.0001-$ i.e., the probability that the results happened by chance., namely 1 in 10,000).

In this repository we found high degrees of parallel changes and a direct correlation between parallel changes and faults. We believe that this repository serves well to adequately evaluate the utility and effectiveness of the methods, techniques and tools that detect interference between parallel changes.

[12] and [13] focused on textual conflict. It showed that only $3 \%$ of the deltas made within 24 hours by different developers physically overlap another's change. The ineffectiveness of textual conflict detection is one of the major reasons to develop a semantic level interference detection algorithm and conduct empirical studies of its effectiveness using industrial/historical data. 


\subsection{Implementation Issues}

In [21] and [22], there are two distinct analyses of semantic interference provided: between adjacent versions and between non-adjacent versions. In this study, we implemented and evaluated the adjacent analysis. The non-adjacent analysis needs an extra assumption: the second change should start from a tested and accepted version. According to our knowledge about the $5 \mathrm{ESS}^{\mathrm{TM}}$ history, this is difficult to guarantee and may not be feasible in practice. To make our study as sound as possible, we used the adjacent analysis that does not require that assumption.

From an analysis on the 5ESS ${ }^{\mathrm{TM}}$ code as well as our personal industrial experience, it is clear that industrial projects make significant use of pointers. Because of this we extended our interference analysis one step further to that of de-referenced variables. We consider this still to be a form of "direct" semantic interference because we are not doing pointer analysis as such but still focusing on def-use pairs to determine interference. Given the efficiency of local analysis and the avoidance of pointer analyses, we believe it is a useful trade-off. The results of our study support that claim

The implementation of the data dependency calculation and program slicing is based on GrammaTech's CodeSurfer [1]. For pointer analysis, we select CodeSurfer's option that distinguishes individual fields in a referenced structure. SCA uses the most precise pointer analysis Codesurfer offers. The $\mathrm{C}$ compiler is Visual C++ 6.0. For the language constructs that do not conform to ANSI C, for example, the macro "\#feature", we made textual changes to the source to pass the compilation. Our preprocessing does not change the semantics of the studied programs.

The study is done on a Pentium III $800 \mathrm{MHz}$ PC with 256M RAM and Microsoft Windows 2000.

\section{Study and Results}

From the observation and implications from the previous study, we propose 3 hypotheses in this evaluation:

1) H1: semantic interference is more likely in higher degrees of parallel changes.

2) H2: semantic interference is a useful predictor of faults in high degree parallel changes.

3) H3: semantic interference detection is lightweight and efficient.

We prepared three sets of changes that represent these different degrees of parallelism. We ran the semantic conflict analyzer on each set. We compared the results from the three sets to evaluate the effectiveness of the detection algorithm on different degrees of parallel changes. We also estimated the overhead by considering the execution time consumed in running the analyzer.

Our study has 5 steps. We introduce the results and their analyses according to the steps.

\subsection{Sample Versions of Different Parallel Degrees}

In this step, we prepared changes to be studied. To supply changes of differing degrees of parallelism, we constructed three equivalent sets of parallel changes from the change and version histories:

1) For the control set, we randomly selected versions that have no parallel changes with respect to a particular release - that is, the interval between the versions are so long, greater than 1 month, that they can not be viewed as a parallel changes.

2) For the low degree of parallelism set, we randomly selected versions that are logically parallel with a reasonable interval of time (from 1 week to 1 month). In this case, we claim the developers have sufficient time to understand the implications of the changes made by others.

3) For the high degree of parallelism set, we randomly selected versions that are logically parallel with a very short interval time, less than 1 week. In this case, it is difficult, we claim, for the developers to fully understand the changes made by others in such a short time ${ }^{1}$.

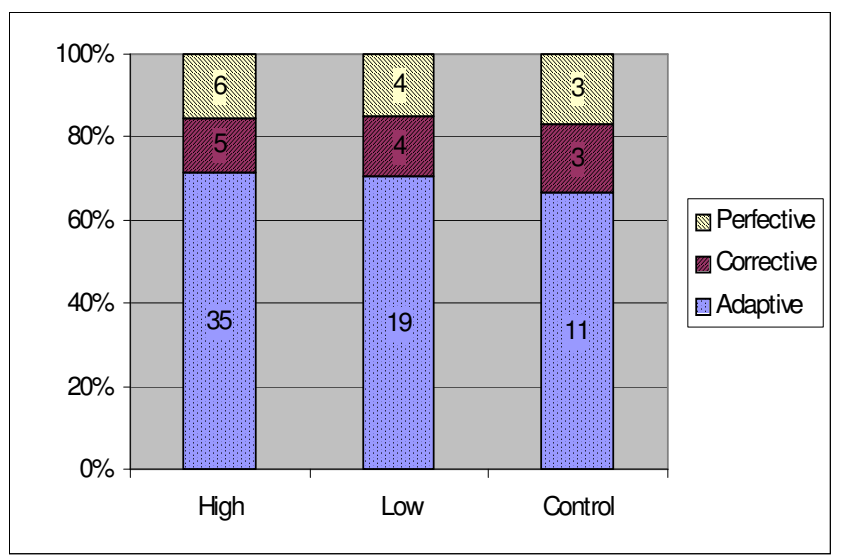

Figure 3 Distribution of the purposes of changes in the three sets. In the High, Low and Control set, the composition of Perfective, Corrective and Adaptive changes are very similar.

\footnotetext{
${ }^{1}$ Our choice of less than a week, between a week and a month, and greater than a month was based the observations in [13] about the phenomena of parallel changes.
} 


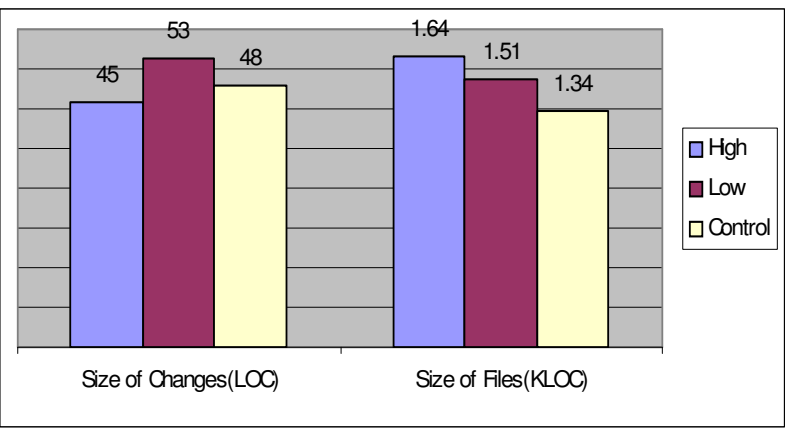

Figure 4 Average sizes of changed lines and files in the three sets. The size of changes is in LOC (Lines Of Code) and the size of file is in KLOC (Kilo- Lines Of Code). The size of changes and files are similar for High, Low and Control sets.

To maximize internal validity, we sample versions for the three sets with nearly identical distribution of different change purposes (adaptive, corrective, and perfective), average size of changes (in number of changed lines), and average size of the source file (in lines of code, LOC). For the sampled versions in the three sets, the composition of the three sets is shown in Figure 3, and the average size of changes and average size of the source file are shown in Figure 4.

\subsection{Calculate Semantic Interference}

In each set of parallel versions, we run SCA to detect the semantic interference between versions. The results are in Table 1.

To quantify the relationship between semantic interference and parallelism, we calculate the Density of Interference and the Frequency of Change for each set.

\section{Density of Interference $=$ detected interferences versions / \# of versions; \\ Frequency of Change $=1$ / Avg. change interval}

The result in Figure 5 shows that the Density of Interferences increases according to the increase of the Frequency of Changes. This supports Hypotheses 1: semantic interference is more likely where there are high degrees of parallelism. This result is congruent

\begin{tabular}{|c|c|c|c|}
\hline Set & Versions & $\begin{array}{c}\text { Interference } \\
\text { versions }\end{array}$ & $\begin{array}{c}\text { Avg. change } \\
\text { interval } \\
\text { (days) }\end{array}$ \\
\hline High & 46 & 19 & 2.3 \\
\hline Low & 27 & 8 & 18.4 \\
\hline Control & 17 & 2 & 265.1 \\
\hline
\end{tabular}

Table 1 Detected interferences in the three sets.

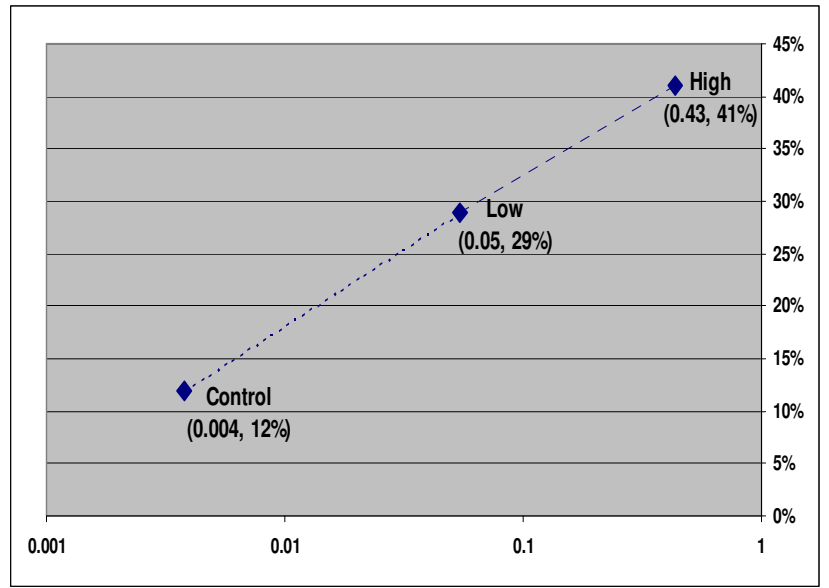

Figure 5 Density of Interference and Frequency of Changes in the three sets. $\mathrm{X}$-axle: Frequency of Changes in changes per day. Y-axle: Density of Interference in interferences per version.

with our earlier findings in with respect to the degree of concurrency and the likelihood of faults [12] [13].

This result also supports for our belief that more conflicts happened at semantic level than at textual level. In the previous study, in high degree parallel changes, only $3 \%$ interference can be detected in the textual level. And the results in Table 1 show that, compared with textual interference, semantic level analysis can disclose significantly more interference than the textual analysis.

\subsection{Identify Related Faults}

Semantic interference by itself does not indicate a fault. The interference might well be intended to correct a fault or to add new processing, etc. It is unintended interference that is likely to represent a fault. Using semantic interference as a predictor of faults, some interferences represent faults while others do not (i.e., are false positives). The critical part of this experiment is to evaluate the effectiveness of the semantic interference in fault prediction.

We use the code fragments that are changed in Corrective MRs to represent faults. For each set of parallel versions (each with its set of semantic interferences), we first mine the change management history to look for Corrective MRs subsequent (in time) to and dependent on these versions with interference. Then we mine the version management system to get the changed code fragments in these Corrective MRs. We note that a logistic regression analysis of our data shows that the high set is significantly different from the control set $(\mathrm{p}<.001)$. 


\subsection{Evaluate SCA's Effectiveness}

The effectiveness of the detection algorithm is based on the match between semantic interference and faulty code. We checked the accuracy of the predictions by checking the defect MRs written against those versions with interference. We also studied the semantic properties of our evaluation results. We divide the evaluation into three sections: 1) where SCA detects semantic interference and there is a fault, and 2) SCA detects interference and there is no fault, or 3) where there is a fault but no detected interference.

\subsubsection{Match: Semantic Interference $\&$ Faults}

For each of the three sets, we compare the semantic interference source code fragments obtained in Section 4.2 with the faulty source code fragments determined in Section 4.3. We classify the results into 3 groups:

- Hit - detected interferences found in faulty code fragments;

- $\quad$ False positive - detected interferences that have no faulty code fragments associated with them;

- $\quad$ Miss - faulty code fragments that have no detected interferences associated with them.

Table 2 shows the results of matching semantic interferences with faults. To fairly compare SCA's effectiveness in the three sets, we calculate the Density of Fault (= Fault-related changes / \# of versions) and the hit ratio (= Hits / Interferences) for each of the three sets. Figure 6 shows that the hit ratio in high degree parallel changes is much higher than that in low degree parallel changes, although their fault density is very similar.

\subsubsection{Semantic Analysis on Matching Results}

Besides the comparisons above, we also analyzed the semantic properties of the hits, misses, and false positives groups in Table 2 according to the classification of errors found in [20].

1) In hit group where the detected interference is found in the faulty code, all the 10 matched interferences are non-pointer variable faults.

$<\quad i=$ pos_no;

$>\quad i=$ pos_not+;

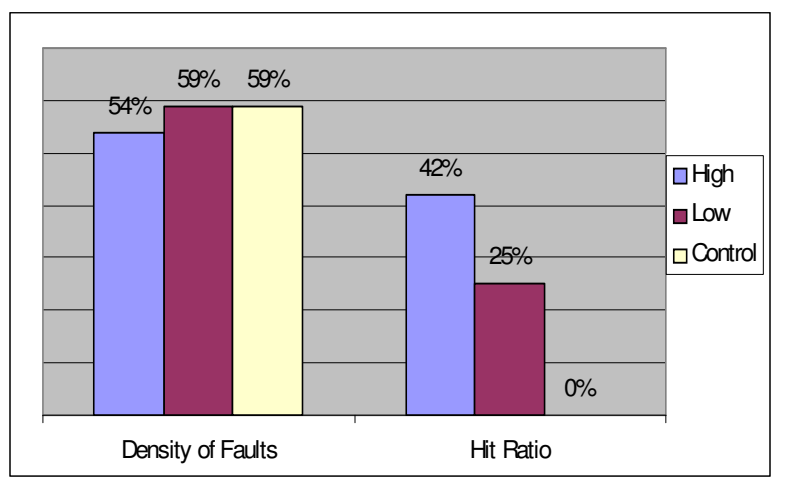

Figure 6 In the three sets, the density of faults is very similar, while the hit ratio in fault prediction is quite different.

In a more specific error classification, all of them are incorrect variable used faults. Eight of the 10 faults are path selection faults. This means an error in variable usages represents a fault in the computation where the program selected a wrong path. Two of the 10 are computation faults, which mean the incorrect variable usage generates erroneous outputs.

2) In false positive group, semantic interferences are detected but are not matched with any faults. Because the noise level is critical for a static analysis tool like SCA, we give a further classification on the 19 false positives:

a) Eight of the 19 are variable or type renaming cases. For example,

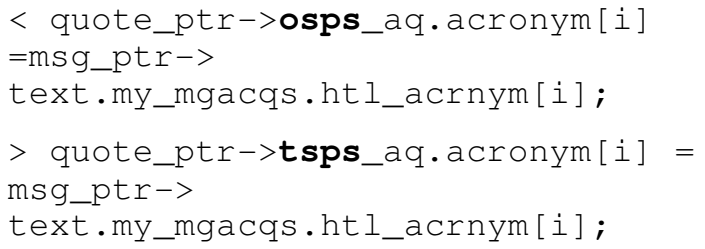

b) Five of the 19 are fault-fixing cases

This kind of semantic interference is intentionally introduced to fix a fault.

c) Three of the 19 are false identification of changes. For example,

\begin{tabular}{|c|c|c|c|c|c|c|}
\hline Sets & Versions & Fault related & Interference & Hit & False positive & Miss \\
\hline High & 46 & 25 & 19 & 8 & 11 & 17 \\
\hline Low & 27 & 16 & 8 & 2 & 6 & 14 \\
\hline Control & 17 & 10 & 2 & 0 & 2 & 10 \\
\hline Total & 90 & 51 & 29 & 10 & 19 & 41 \\
\hline
\end{tabular}

Table 2 Match the detected semantic interference fragments with faulty codes. 


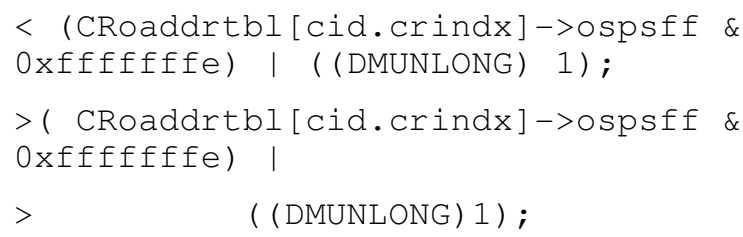

This kind false positive is an artifact of CodeSurfer and comes from the incorrect identification of same vertex in two versions. In this detection algorithm, the corresponding vertex in the two versions is identified by its type and the associated text. So, in the change above, we view the two statements as different, although only spaces were added in the second version. We did not use semantic equivalence evaluation as found in [24] because it is too expensive to compute in a real project.

3) The miss group shows the fault-related versions that are invisible to our interference detection algorithm. There were 17 faults that were invisible - i.e., that were undetected by SCA. We classify these invisible faults according to their semantic properties. This can guide users to utilize our interference detection approach in effective ways.

a) Eight of the 17 are control flow faults.

$<$ if $(i<5)$
$>$ if $(i<=5)$

For such kind of change, no impact happened on variable definition and use. So our interference detection algorithm cannot identify such faults that are related to control flow errors.

b) Five of the 17 are faults related to pointer variables.

We reviewed the sampled versions and found some pointer arithmetic operations changed the target objects of pointers. But such changes are ignored in our algorithm because Codesurfer assumes that pointer arithmetic will not change the pointer-to set.

c) Four of the 17 are basically other faults which can not be categorized into the error classification in [20].

In summary, the match between semantic interferences and predicted faulty code supports Hypothesis H2. Moreover, the semantic analysis on false positives also confirms that SCA suffers the same problems as many other static analysis tools. And for the non-pointer variables that static analysis can give a more precise analysis than for the pointers, SCA can also give a more precise prediction of potential faults.

\subsection{Evaluate SCA's Efficiency}

While evaluating the efficiency of SCA, we use the time used in calculating semantic interference as the overhead. In analyzing the semantic interference on the sample versions, the average time is about 2 minutes. In this overhead, $83 \%$ is spent on the program dependency analysis with CodeSurfer, and the time for the calculating and detecting interferences is only $17 \%$ of the overhead.. And the average time to compile the analyzed version is 1.8 minutes. This result supports Hypothesis H3: semantic interference is a lightweight and efficient fault prediction approach.

Based on these results, we can claim that the use of SCA is both effective and efficient and will aid developers in finding and fixing faults. As soon as a developer finishes a new version, SCA can give instant feedback about conflicts. The immediate warnings can remind developers to check or fix faults in the new version while they are still familiar with what changes have been made in this new version as well as the reasons for making them. For the versions we studied, it is usually 150 days on average after the faultinducing changes were made when they were found by various means such as testing, etc. The use of SCA will save the effort of having to rediscover or recall what was done 5 months earlier.

\section{Validity Analysis}

To analyze the soundness of this experiment, we discuss its construct, internal, external validity, and conclusion validity.

We focus on a specific and well-defined form of semantic interference between versions. Given that there are multiple levels of parallelism in a large-scale development, we feel that our distinction between high, low and no degrees of parallelism is justified for this study evaluating the efficiency and effectiveness of our analyzer. We also claim the delay time construct is well defined and justified as well: the time from the version commit until the opening of the fault MR. The problematic time construct is the fix time. First, one should view that as a maximum possible time where only a portion of that time is actually spent finding and fixing the fault. Second, only a portion of the actual time is spent in finding the problem and rediscovery; it is this time that would be saved by our analyzer.

In evaluating the effectiveness and efficiency of the interference detection algorithm, we used the comparisons among sets of different degrees of parallelism. To filter out factors other than parallelism, we have checked the similarity among the sets in the distribution of changes of different purposes, the file 
sizes, the change sizes, and the density of fault-related changes. We argue that the equivalence of these factors rules out confounding variables.

We believe that our results our consistent with what one would intuitively expect about parallel changes and what is supported with our earlier studies: highly parallel changes do not allow time for developers to adequately understand the implications of changes and hence are more prone to faults as a result of these changes. Our current results are consistent with our earlier results: there is a significant correlation ( $p$ $<.001$ ) between the degrees of parallelism, semantic interferences and faults. The new and interesting results here (see Figure 6) also agree with ones intuition about adaptive changes: there are likely to be more interfering changes made to add new functionality than in correcting faults, or improving existing functionality.

Although our study is based on the history data in a pessimistic version control system, SCCS, this approach can be easily extended to optimistic version control system, such as Concurrent Versions System (CVS), which is widely used in open source projects. CVS can supply the same kinds of data as SCCS for our semantic interference detection algorithm. The only variation in the evaluation process is the use of nonadjacent versions of the interference detection algorithm. This is because, in CVS, the sequential order introduced by the check-in time in SCCS will not be valid.

A threat to our study is that $5 \mathrm{ESS}^{\mathrm{TM}}$ is a very largescale real-time project with a large number of developers, geographically distributed. We argue, however, that the subsystem we studied is perhaps by itself more representative of a typical large project. The critical factor, however, is the issue of parallel changes to the same files by different people - i.e., feature ownership rather than code ownership. This form of development is becoming more prevalent, and this supports our claim for external validity.

On the basis of a sufficient set of versions to give us a reasonably good level of statistical power, appropriate factoring and precision, and reasonable reliability in the tools we use (e.g., see [9]), we believe that we have strong conclusion validity.

\section{Related Work}

Program slicing is an important technique in analyzing properties of programs. [6] proposed the combination of a program dependency graph and program slicing to provide conflict detection. And Yang [24] increased the soundness of semantic conflict detection by semantic preserving transformations. Different from them, SCA focuses only on variable defuses rather than the whole dependency graph. While such a simplification means we do not catch all possible faults, it does make SCA a lightweight tool that is both feasible and effective in real projects. The results of Step 5, the efficiency evaluation of the detection algorithm, give strong support for our simplification.

[15], [17], and [19] propose change impact analysis based on atomic change classifications and associate them with test cases. They work at the method level, and compare two abstract syntax trees, thus providing more precision but paying a higher overhead. SCA works at the statement level, comparing the vertices by variable def-uses and associated text. Ours is more narrowly focused on significantly less overhead.

[18] uses fault localization to identify changes from the version management system and a fault database, and correlate them as fault-inducing changes. However, our approach is based on the semantic analysis on the changes and their interference, while [18] focuses on relating the events in version histories with fault databases.

Passed and failed test cases are used in [25] to filter out non-related test cases and to select possible faultinducing changes. Their work focuses on the localization of fault-inducing changes by running test cases, while our approach focuses on the prediction of faults with static analysis of changes that semantically interfere.

Program chopping [5] can minimize possible faultinducing code fragments. Compared with static program slicing we use, dynamic slicing can improve the precision for pointer analysis and reduce false positives in semantic interference detection. However executable versions are required beforehand. This imposes a significant build overhead in any large system.

In the empirical studies with version control repositories, Atkins et al. [2] uses change history and an effort estimation model [4] to calculate the effort that has been saved using the Version Editor (VE). This study illustrates an important point: the versions found in the repository were separated into two groups based on a criterion that was useful in the empirical study and a significant result was obtained on the basis of this differentiation. The quantitative results provide evidence showing the usefulness of the software tool.

However, the origin of the data in our study is quite different from theirs. In the construction of the control 
and treatment groups, they were able to differentiate the historical data into $\mathrm{VE}$ and non-VE related groups. Without that extra-repository distinction (i.e., the VE footprint instrumented into the histories), the evaluation would not have been possible. Thus, while this empirical study did use historical data from version and change management, it was, in a real sense, instrumented data. While in our evaluation of SCA, all the data in our study was not instrumented, but data readily available in the repositories.

[3] and [26] predict faults by mining change histories. But [3]'s granularity is larger than ours: the number of changes on a file, or the number of lines changed in a period of time. They do not consider interference between changes, whether at the textual level or the semantic level. [26] focuses on structurerelated entities, such as fields or functions in a file, or files in a directory, and predicts faults from the incomplete changes. We, on the other hand, focus on the semantics of the code, predicting faults from semantic interference.

\section{Conclusions and Future Work}

Our research has yielded two important contributions: first, we have shown that a limited form of semantic interference detection can provide an effective and useful means of predicting faults in an increasingly common context; and second, we created an effective and novel design for rigorous experimental evaluation of analysis tools using and mining change and version management repositories.

While our technique is incomplete both in detecting semantic interference and in predicting faults, it is both efficient (taking roughly the time of a compilation) and effective in predicting faults. Despite our tool suffering the same problems as many other static analysis tools (i.e., unsoundness due to the imprecision of pointer analysis), the resulting analyses and predictions are still extremely useful in directing developers' attention to potential faults.

The results of our evaluative experiment are as follows:

1) Semantic interference is significantly higher in high group (twice the other two);

2) Semantic interference detection is effective in predicting non-pointer variable faults from the detected interferences in versions changed within short time periods;

3) Of the detected semantic interferences, $40 \%$ in the high group matched with known faults, $25 \%$ in the low group (33\% in both);
4) The overhead of using our semantic interference detection approach is very low and can help developers to find faults very early; and,

5) Preciseness of pointer analysis, identification of variable renaming, and control-flow changes are the major factors that affect the effectiveness in detecting interference and predicting faults.

Our experimental design itself is a significant contribution to providing rigorous evaluation of tools. We avoid the invalidity problems of contrived faults. Change and version management repositories provided a large enough population to obtain a variety of sample data such as change purpose, size of changed code, and size of source file. By classifying the sampled data according to the degree of parallelism, we constructed a control group and two treatment groups.

To provide an effective evaluation, the fault sets were mined from the version and change management repositories, rather than intentionally introduced. This "mundane realism" not only removes the internal validity problems associated with fault seeding (the representativeness of the faults seeded, the placement of those faults, and the frequency of fault occurrence, etc), but also increases the external validity or the study.

The results from our study also suggest ways of combining our approach with that of others to improve the effectiveness of the semantic interference detection algorithm. As complementary approaches to static analysis tools such as SCA, dynamic analysis techniques, such as dynamic slicing [5] or symbolic execution [7], can identify control flow dependencies and analyze dereferences with more precision. And light weight compilation techniques, such as Island Grammars [10] [11], can reduce the workload for semantic analysis by eliminate the requirement on compilation.

\section{Acknowledgements}

We thank Harvey Siy, University of Nebraska, Omaha, for his help on the change management system of 5ESS ${ }^{\mathrm{TM}}$ and his suggestion about Island Grammars; Barbara Ryder, Rutgers University, for her review of our preliminary results; and Xiangyu Zhang, Purdue University, for the discussion on dynamic slicing issues. This work was supported in part by NSF CISE Grant IIS-0438967.

\section{References}

[1] P. Anderson, and T. Teitelbaum, "Software Inspection Using CodeSurfer", Proc. of the First Workshop on Inspection in Software Engineering (WISE'01), Paris, France, July 2001, 4-11. 
[2] D. Atkins, T. Ball, T. Graves, and A. Mockus. "Using version control data to evaluate the impact of software tools: A case study of the version editor", IEEE Transactions on Software Engineering, Vol. 28, No. 7, July 2002, 625-637.

[3] T.L. Graves, A.F. Karr, J.S. Marron and H. Siy, "Predicting Fault Incidence Using Software Change History," IEEE Transactions on Software Engineering, Vol. 26, No. 7, July 2000. 653-661.

[4] T.L. Graves, A. Mockus, "Inferring Change Effort from Configuration Management Databases", Pro. of the Fifth International Symposium on Software Metrics, IEEE, 1998, 267-273.

[5] N. Gupta, H. He, X. Zhang, and R. Gupta, "Locating Faulty Code Using Failure-Inducing Chops", Proc. of the 20th IEEE/ACM International Conference on Automated Software Engineering (ASE 2005), Long Beach, California, November 2005, 263-272.

[6] S. Horwitz, J. Prins, and T. Reps, "Integrating noninterfering versions of programs", $A C M$ Transactions on Programming Languages and Systems, Vol. 11, No. 3, July 1989, 345-387.

[7] S. Khurshid, C. Pasareanu, and W. Visser, "Generalized Symbolic Execution for Model Checking and Testing", Proc. of the 9th International Conference on Tools and Algorithms for Construction and Analysis of Systems (TACAS 2003), Warsaw, Poland, Apr 2003, 553-568.

[8] K. Martersteck, and A. Spencer, "Introduction to the 5ESS ${ }^{\text {TM }}$ Switching System", AT\&T Technical Journal, Vol. 64, No. 6, part 2, July-August 1985, 1305-1314.

[9] A. Mockus, and L.G. Votta, "Identifying Reasons for Software Changes Using Historic Databases", Proc. of IEEE International Conference on Software Maintenance (ICSM'00), San Jose, CA, USA, October. 2000, 120-130.

[10] L. Moonen, "Generating Robust Parsers Using Island Grammars", Proc. of the Eighth Working Conference on Reverse Engineering (WCRE'01), Stuttgart, Germany, October. 2001, 13-22.

[11] L. Moonen, "Lightweight Impact Analysis Using Island Grammars", Proc. of Tenth International Workshop On Program Comprehension (IWPC'02), June 2002, 219-228.

[12] D.E. Perry, and H.P. Siy, "Challenges in Evolving a Large Scale Software Product", Proc. of the International Workshop on Principles of Software Evolution, the 20th International Software Engineering Conference, Kyoto, Japan, April 1998, 251-260.

[13] D.E. Perry, H.P. Siy, and L.G. Votta, "Parallel Changes in Large Scale Software Development: An Observational Case Study", ACM Transactions on Software Engineering and Methodology, Vol. 10, No. 3, July, 2001, 308-337.

[14] R. Purushothaman, and D.E Perry, "Towards Understanding the Rhetoric of Small Source Code Changes", IEEE Transactions on Software
Engineering, Special Issue on Mining Software Repositories, Vol. 31, No. 6, June 2005, 511-526.

[15] X. Ren, F. Shah, F. Tip, B.G. Ryder, and O. Chesley, "Chianti: A Tool for Change Impact Analysis of Java Programs", Proceedings of the $19^{\text {th }}$ Annual ACM SIGPLAN Conference on Object-Oriented Programming, Systems, Languages, and Applications (OOPSLA 2004), Oct 2004, 432-448.

[16] M.J. Rochkind, "The Source Code Control System", IEEE Transactions on Software Engineering, Vol. SE-1, No. 4, December 1975, 364-370.

[17] B.G. Ryder, and F. Tip, "Change impact analysis for object-oriented programs", Proc. of the 2001 ACM SIGPLAN-SIGSOFT workshop on Program analysis for software tools and engineering, June 2001, Snowbird, Utah, 46-53.

[18] J. Sliwerski, T. Zimmermann, and A. Zeller, "When do changes induce fixes? On Fridays", Proc. of International Workshop on Mining Software Repositories (MSR), Saint Louis MO, May 2005.

[19] M. Stoerzer, B.G. Ryder, X. Ren, and F. Tip, "Finding Failure-Inducing Changes using Change Classification", Research Report RC 23729, IBM, September 2005.

[20] K. Tewary and M.J. Harrold, "Fault Modeling using the Program Dependence Graph", International Symposium on Software Reliability Engineering, November 1994, 126-135.

[21] G.L. Thione, "Detecting Semantic Conflicts in Parallel Changes", MSEE Thesis, The Department of Electrical and Computer Engineering, The University of Texas at Austin, December 2002. 98pp.

[22] G.L. Thione, and D.E. Perry, "Parallel Changes: Detecting Semantic Interferences", The 29th Annual International Computer Software and Applications Conference (COMPSAC 2005), Edinburgh, Scotland, July 2005, 47-56.

[23] P.A. Tuscany, "Software development environment for large switching projects", Proc. of Software Engineering for Telecommunications Switching Systems Conference, 1987.

[24] W. Yang, S. Horwitz, and T. Reps, "A program integration algorithm that accommodates semanticspreserving transformations", ACM Transactions on Software Engineering and Methodology, Vol. 1, No. 3, July 1992, 310-354.

[25] A. Zeller, "Yesterday, my program worked. Today, it does not. Why?" Proc. of Joint 7th European Software Engineering Conference (ESEC) and 7th ACM SIGSOFT International Symposium on the Foundations of Software Engineering (FSE-7), Vol. 1687 of LNCS, Toulouse, France, September 1999, 253-267.

[26] T. Zimmermann, P. Weibgerber, S. Diehl, A. Zeller, "Mining Version Histories to Guide Software Changes", Proc. of the 26th International Conference on Software Engineering (ICSE 2004), Edinburgh, UK, May 2004, 563-572 